\title{
Counting RNA pseudoknotted structures (extended abstract)
}

\author{
Cédric Saule $^{1,4}$, Mireille Régnier ${ }^{4,2}$, Jean-Marc Steyaert ${ }^{2,4}$, Alain Denise ${ }^{1,3,4}$ \\ ${ }^{1}$ LRI, Université Paris-Sud and CNRS, bât. 490, 91405 Orsay cedex, France \\ ${ }^{2}$ LIX, Ecole Polytechnique and CNRS, 91128 Palaiseau cedex, France \\ ${ }^{3}$ IGM, Université Paris-Sud and CNRS, bât. 400, 91405 Orsay cedex, France \\ ${ }^{4}$ INRIA Saclay, Parc Orsay Université, 4 rue Jacques Monod, 91893 Orsay cedex, France
}

\begin{abstract}
In 2004, Condon and coauthors gave a hierarchical classification of exact RNA structure prediction algorithms according to the generality of structure classes that they handle. We complete this classification by adding two recent prediction algorithms. More importantly, we precisely quantify the hierarchy by giving closed or asymptotic formulas for the theoretical number of structures of given size $n$ in all the classes but one. This allows to assess the tradeoff between the expressiveness and the computational complexity of RNA structure prediction algorithms.

Résumé. En 2004, Condon et ses coauteurs ont défini une classification des algorithmes exacts de prédiction de structure d'ARN, selon le degré de généralité des classes de structures qu'ils sont capables de prédire. Nous complétons cette classification en y ajoutant deux algorithmes récents. Chose plus importante, nous quantifions la hiérarchie des algorithmes, en donnant des formules closes ou asymptotiques pour le nombre théorique de structures de taille donnée $n$ dans chacune des classes, sauf une. Ceci fournit un moyen d'évaluer, pour chaque algorithme, le compromis entre son degré de généralité et sa complexité.
\end{abstract}

Keywords: bioinformatics, RNA structures, pseudoknots, enumeration, bijective combinatorics

\section{Introduction}

In bioinformatics, the RNA structure prediction problem consists, given a RNA sequence, in finding a conformation that the molecule is likely to take in the cell. In [3], Condon and coauthors classified RNA structure prediction algorithms according to the inclusion relations between their classes of structures. The class of structures of a given algorithm is the set of structures that can be, in theory, returned by the algorithm. Condon et al. focused only on exact algorithms, that is algorithms that guarantee to give an optimal solution to the structure prediction problem, stated as an optimisation problem. They considered the class of pseudoknot-free structures [13, 25] (PKF), and the following classes for pseudoknotted structures: Lyngs $\emptyset$ and Pedersen (L\&P) [11], Dirks and Pierce (D\&P) [4], Akutsu and Uemura (A\&U) [1, 20], and Rivas and Eddy (R\&E) [16]. They notably proved the following inclusion relations: $P K F \subset L \& P \subset D \& P \subset A \& U \subset R \& E$. Since then, two other exact prediction algorithms have been developed, involving new classes: Reeder and Giegerich $(R \& G)$ [15] and Cao and Chen (C\&C) [2] algorithms.

1365-8050 @ 2010 Discrete Mathematics and Theoretical Computer Science (DMTCS), Nancy, France 
In this paper, we aim to quantify the tradeoff between the computational complexity and the expressiveness of all these algorithms. For this purpose, we compare them from the double point of view of their computational complexity and the cardinality of their class of structures, for a given size $n$. And we give closed or asymptotic formulas for the theoretical number of structures of given size $n$ except for the class $R \& E$. More precisely, we establish that, except for the $L \& P$ class whose asymptotic formula is simpler, the number of structures of size $n$ is, asymptotically, $\frac{\alpha}{2 \sqrt{\pi} n^{3 / 2}} \omega^{n}$, where $\alpha$ and $\omega$ are two constants which depend of the class. Additionally, we place the two new classes, $\mathrm{R} \& \mathrm{G}$ and $\mathrm{C} \& \mathrm{C}$, in Condon et al's hierarchy. The following table summarizes our results. We indicate by "*" the classes that had not been enumerated before. The class "All" denotes the whole set of pseudoknotted structures. The row "Compl" gives the complexity of each algorithm.

\begin{tabular}{|l|c|c|c|c|c|}
\hline Class & asympt. & $\alpha$ & $\omega$ & Compl. & Remark \\
\hline PKF & $\frac{\alpha}{2 \sqrt{\pi} n^{3 / 2}} \omega^{n}$ & 2 & 4 & $\mathcal{O}\left(n^{3}\right)$ & Catalan numbers \\
\hline L\&P $*$ & $\frac{1}{2} \omega^{n}$ & - & 4 & $\mathcal{O}\left(n^{5}\right)$ & Closed formula \\
\hline C\&C $*$ & $\frac{\alpha}{2 \sqrt{\pi} n^{3 / 2}} \omega^{n}$ & 1,6651 & 5,857 & $\mathcal{O}\left(n^{6}\right)$ & \\
\hline R\&G $*$ & $\frac{\alpha}{2 \sqrt{\pi} n^{3 / 2}} \omega^{n}$ & 0,1651 & 6,576 & $\mathcal{O}\left(n^{4}\right)$ & \\
\hline D\&P $*$ & $\frac{\alpha}{2 \sqrt{\pi} n^{3 / 2}} \omega^{n}$ & 0,7535 & 7,315 & $\mathcal{O}\left(n^{5}\right)$ & \\
\hline A\&U $*$ & $\frac{\alpha}{2 \sqrt{\pi} n^{3 / 2}} \omega^{n}$ & 0,6575 & 7,547 & $\mathcal{O}\left(n^{5}\right)$ & \\
\hline R\&E & open & - & - & $\mathcal{O}\left(n^{6}\right)$ & \\
\hline All & $\sqrt{2} \cdot 2^{n} \cdot\left(\frac{n}{e}\right)^{n}$ & - & - & NPC & Involutions with no fixed points \\
\hline
\end{tabular}

A number of works have been done on combinatorial enumeration of RNA structures without pseudoknots, see e.g. [24, 21, 7, 12, 10] or, more recently, with pseudoknots, as in [22, 17, 8, 9] for instance. Our purpose is different, as our classes of structures are not defined per se, but correspond to given exact prediction algorithms.

The paper is organised as follows. In Section 2, we give some notation and definitions. In Section 3, we present a bijection between the L\&P class and a class of planar maps, leading to a closed formula for the $L \& P$ class. In Section 4, we establish that each of the classes D\&P, A\&U, R\&G, C\&C, and L\&P can be encoded by a context-free language. For each of them, we derive an equation for the generating function, leading to an asymptotic formula for the number of structures of size $n$. In Section 5, we conclude by giving some remarks on the expressiveness of the structure prediction algorithms compared to their complexity.

\section{Definitions and notation.}

A RNA secondary structure (possibly with pseudoknots) is given by a sequence of integers $(1,2, \ldots, n)$ and a list of pairs $(i, j)$, called basepairs or arcs, where $i<j$ and each number in $\{1,2, \ldots, n\}$ appears exactly in one pair. Such a structure can be represented as in Figure 11, where each basepair $(i, j)$ is represented by an edge between $i$ and $j$. In real RNA structures there are unpaired bases, but we do not consider them.

Definition 1 (Crossing arcs) Let $(i, j)$ and $(k, l)$ two arcs such that $i<k$. We say that $(i, j)$ and $(k, l)$ are crossing if $i<k<j<l$. 
Definition 2 (Crossing graph) The crossing graph of an RNA structure is a graph $G$ defined as follows: the vertices of $G$ are the arcs of the structure, and two vertices of $G$ are connected by an edge if and only if their two corresponding arcs are crossing.

Definition 3 (Pseudoknot) A pseudoknot is a set of arcs that is not a singleton and that corresponds to a maximal connected component in the crossing graph.

Definition 4 (Simple pseudoknot [1]) A pseudoknot $P$ is simple if there exist two numbers $j_{1}$ and $j_{2}$, with $j_{1}<j_{2}$, such that: (i) each arc $(i, j)$ in P satisfies either $i<j_{1}<j \leq j_{2}$ or $j_{1} \leq i<j_{2}<j$, (ii) and if two arcs $(i, j)$ and $\left(i^{\prime}, j^{\prime}\right)$ satisfy $i<i^{\prime}<j_{1}$ or $j_{1} \leq i<i^{\prime}$, then $j>j^{\prime}$.

The first property ensures that, for each arc of $P$, one of its ends exactly is between $j_{1}$ and $j_{2}$. And the arcs are divided in two sets: those having their other end smaller than $j_{1}$, and those having their other end greater than $j_{2}$. We call these two sets, respectively, the left part and the right part of the pseudoknot. The second property of the definition ensures that two arcs in the same set cannot intersect each other. Figure 1 shows a simple pseudoknot.

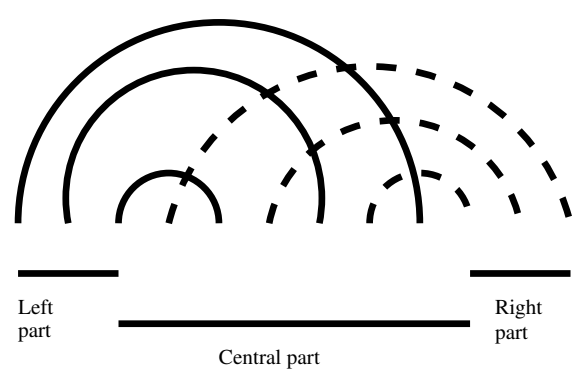

Fig. 1: A pseudoknot given by the sequence $(1,2, \ldots, 12)$ and the $\operatorname{arcs}(1,9),(2,7),(3,5),(4,12),(6,11),(8,10)$. This pseudoknot is simple, with $j_{1}=4$ and $j_{2}=9$.

Definition 5 (H-type Pseudoknot) A H-type pseudoknot is a simple pseudoknot having the following additional property: each arc in one of the two above sets crosses all the arcs of the other set.

\section{A bijection between the L\&P structures and a class of planar maps.}

The Lyngsø-Pedersen (L\&P) class is the simplest class of pseudoknotted structures. According to [11] and [3], a structure is in the L\&P class if and only if it contains either no pseudoknot or a unique H-type pseudoknot, and this pseudoknot is not embedded under any arc. Between any two consecutive ends of the arcs of the pseudoknots, there can be a nested structure. Theorem 11, and its straightforward Corollary 1. give the closed formula and the asymptotic formula for the number of such structures, respectively.

Theorem 1 The number of $\mathrm{L} \& \mathrm{P}$ structures with $\mathrm{n}$ arcs is:

$$
L P(n)=\frac{1}{2} \cdot 4^{n}-\left(\begin{array}{c}
2 n+1 \\
n
\end{array}\right)+\left(\begin{array}{c}
2 n-1 \\
n-1
\end{array}\right)+\frac{1}{n+1}\left(\begin{array}{c}
2 n \\
n
\end{array}\right) .
$$




\section{Corollary 1}

$$
L P(n) \sim \frac{1}{2} \cdot 4^{n} .
$$

\section{Proof of Theorem 1;}

The proof is bijective: we establish a bijection between the set of L\&P structures of any size $n$ and the set of rooted isthmusless planar maps with $n$ edges and one or two vertices. The first two terms of the formula count the number of such maps with two vertices [18, 23], while the last term, a Catalan number, counts the number of such maps with one vertex [19]. Hence the theorem.

A planar map is a proper embedding of a connected planar graph. It is said isthmusless if the deletion of any edge does not split the graph. A rooted planar map is a planar map where a vertex and an edge adjacent to it are distinguished. Any planar map with $n$ edges can be represented by two permutations $\sigma$ and $\tau$ on $\{+1,-1,+2,-2, \ldots,+(n-1),-(n-1),+n,-n\}$, in the following way: The edges of the map are numbered from 1 to $n$. Then, for any edge $i$, one labels its extremities with $+i$ and $-i$, respectively. By convention, the root edge is labelled with +1 and -1 , in such a way that -1 labels the extremity adjacent to the root vertex. Now, the two permutations are as follows:

- the permutation $\sigma$ is an involution without fixed points that represents the edges of the map. Each cycle of $\sigma$ is of size two and contains both ends of one edge: $\sigma=(+1,-1),(+2,-2), \ldots,(+n,-n)$.

- the permutation $\tau$ has as many cycles as vertices in the map. Each cycle is given by the sequence of labellings around the corresponding vertex, clockwise.

Let us consider a L\&P structure $S$ with n edges, and let us label the left extremities of its arcs with $+1,+2, \ldots,+n$ from left to right, and give to each right foot the label $-i$ if the corresponding left foot has label $+i$. Let $w=\left[w_{1}, w_{2}, \ldots w_{2 n}\right]$ be the sequence of labels of $S$, from left to right. From any $w$ we can now construct two permutations $\sigma$ and $\tau$ that represent an isthmussless rooted planar map with one or two vertices. Regarding $\sigma$, we just set $\sigma=(+1,-1) \ldots(+n,-n)$.

Let us first consider the simple case where there is no crossing in the structure. It is known for a long time that such nested structures are counted by Catalan numbers. This can be established, for example by a folkloric bijection with planar maps having one vertex, by setting $\sigma$ as above, and $\tau=(w)$.

Now suppose that there is a pseudoknot in the structure, and let us present a bijection between the set of such structures and the set of rooted ithmusless planar maps with two vertices. Start from $w$. Since $\tau$ must have two cycles, we have to split $w$ in two parts that will be the two cycles. Let us define the left set (resp. the right set) of arcs of the pseudoknot, respectively, as the set of arcs whose left (resp. right) extremities are in the left (resp. right) part of the pseudoknot, where left and right parts are defined as in Section 2 There are two cases:

Case 1 . There is only one arc in the right set. In this case, let $\ell$ be the position of the first right extremity of an arc in the left set. We cut $w$ between positions $\ell-1$ and $\ell$. Each part corresponds to a cycle of $\tau$ : $\tau=\left(w_{1}, \ldots, w_{\ell-1}\right)\left(w_{\ell}, \ldots, w_{2 n}\right)$. See Figure 2 for an illustration.

Case 2. There are at least two arcs in the right set. We cut $w$ just before the first right extremity of an arc in the right set. See Figure 3

Let us show that, in both cases, the resulting map is planar and isthmusless. At first, remark that if the map is not planar or has an isthmus, necessarily it comes from arcs that are involved in the pseudoknot. Indeed, by construction, non crossing arcs in the structure give non crossing loops in the map. So, without loss of generality, we can consider only structures where all the arcs are involved in the pseudoknot. Consider such a structure with $n$ arcs. In the case 1 , we have $w=[+1,+2, \ldots,+(n-1),+n,-(n-$ 


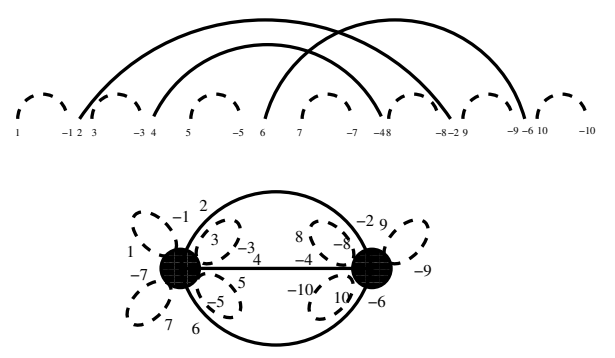

Fig. 2: Top, a L\&P structure corresponding to case 1. Bottom, the corresponding planar map. Arcs not involved in the pseudoknot are drawn in dotted lines.

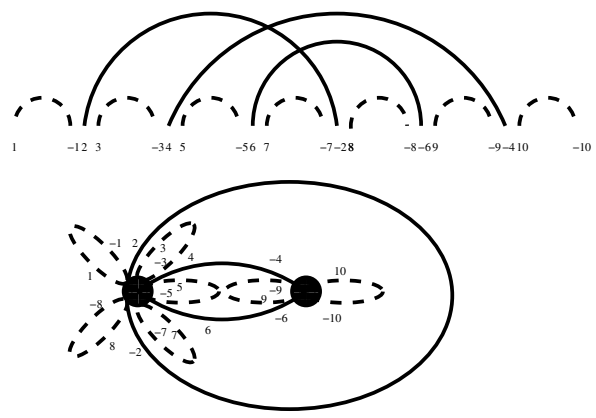

Fig. 3: Top, a L\&P structure corresponding to case 2. Bottom, the corresponding planar map. Arcs not involved in the pseudoknot are drawn in dotted lines.

$1),-(n-2), \ldots,-1,-n]$, hence $\tau=(+1,+2, \ldots,+n)(-(n-1),-(n-2), \ldots,-1,-n)$. Clearly, this gives a planar map, since the two cycles of $\tau$ are in opposite order. And there is no isthmus because all edges go from one vertex to the other. In the case 2 , we have $w=[+1,+2, \ldots,+(\ell-$ $1),+\ell,+(\ell+1), \ldots,+n,-(\ell-1), \ldots,-2,-1,-n,-(n-1), \ldots,-\ell]$, hence $\tau=(+1,+2, \ldots,+(\ell-$ $1),+\ell,+(\ell+1), \ldots,+n,-(\ell-1), \ldots,-2,-1)(-n,-(n-1), \ldots,-\ell)$. Again, this gives a planar map: edges $1,2, \ldots, \ell-1$ are nested loops, and edges $\ell, \ldots, n$ go from one vertex to the other, without any crossing. And there is no isthmus because the number of edges going from one vertex to the other, $n-\ell+1$, is greater or equal to 2 .

Now let us present the converse transformation. Consider an isthmusless rooted planar map with two vertices, given by $\sigma=(+1,-1),(+2,-2), \ldots,(+n,-n)$ and $\tau$ having two cycles. We aim to construct the sequence $w$ that represents the corresponding pseudoknotted structure. Let us consider the cycle of $\tau$ which contains 1 , and write it in such a way that it begins with 1 . Let us call $u$ this sequence of labels. This gives the first part of the sequence $w$. We are now searching for the second part of $w$, that is the sequence $v$ such that $u v=w$. For that purpose, consider the set of isolated labels, that is the labels in $u$ that have not their opposite label in $u$. We have the two following cases:

Case 1. There is no pair $(+i,-i)$ in $u$ such that the isolated labels are located between $+i$ and $-i$. Let $+j$ the penultimate isolated label in $u$. Write the second cycle of $\tau$ in such a way that it begins with $-j$. This gives $v$, and there is exactly one edge in the second part of the pseudoknot. 
Case 2. There is a pair of labels $(+i,-i)$ in $u$ such that all isolated labels are located between $+i$ and $-i$. Let $+j$ the last isolated label in $u$. Write the second cycle of $\tau$ in such a way that it begins with $-j$. This gives $v$. In this case, there are at least two edges in the second part of the pseudoknot.

\section{Asymptotic enumeration of pseudoknotted structures.}

\subsection{A context-free encoding for simple and H-type pseudoknots}

As will be seen farther, all the classes that are involved in exact prediction algorithms but one involve either H-type pseudoknots or simple pseudoknots. The only exception is the R\&E class. Here we define a transformation that allow to encode any class of pseudoknotted structures where all pseudoknots are simple by a context-free language.

Let us first recall some definitions. Let $L$ be a language on a given alphabet $A$, and $w=w_{1} w_{2} \ldots w_{n}$ a word of $L$, where the $w_{i}$ 's are the letters of $w$. A word $v$ is a subword of $w$ if $v=w_{i_{1}} w_{i_{2}} \ldots w_{i_{k}}$, where $1 \leq i_{1}<i_{2}<\ldots<i_{k} \leq n$. The projection of $w$ onto an alphabet $A^{\prime} \in A$ is the subword $w^{\prime}$ obtained by erasing in $w$ all letters that do not belong to $A^{\prime}$. The projection of $L$ onto $A^{\prime}$ is the set of projections of the words of $L$ onto $A^{\prime}$. Finally, let us recall that the Dyck language on any two-letter alphabet $\{d, \bar{d}\}$ is the language of balanced parentheses strings, where $d$ and $\bar{d}$ stand, respectively, for opening and closing parentheses. Now we can state twe two following straightforward lemmas:

Lemma 1 Any class of pseudoknotted structures where all pseudoknots are simple can be encoded by the words of a language $L$ on the alphabet $\{d, \bar{d}, x, \bar{x}, y, \bar{y}\}$ where $(i) d$ and $\bar{d}$ encode, respectively, the left and right ends of arcs that are not involved in pseudoknots; (ii) $x$ and $\bar{x}$ encode, respectively, the left and right ends of arcs that are involved in the left parts of pseudoknots; (iii) $y$ and $\bar{y}$ encode, respectively, the left and right ends of arcs that are involved in the right parts of pseudoknots. Additionally, the projection of the language to the alphabet $\{d, \bar{d}\}$ (resp. $\{x, \bar{x}\},\{y, \bar{y}\}$ ) is a sublanguage of the Dyck language on the same alphabet.

Lemma 2 Let $S$ be a pseudoknotted structure, and $w$ be the word on $\{d, \bar{d}, x, \bar{x}, y, \bar{y}\}$ that encodes $S$. Then every simple pseudoknot in $S$ is encoded by a subword $v$ of $w$, such that $v=x^{n} y^{m_{1}} \bar{x}^{n_{1}} y^{m_{2}} \bar{x}^{n_{2}} \ldots$ $y^{m_{k}} \bar{x}^{n_{k}} \bar{y}^{m}$, where $n_{1}+n_{2}+\ldots+n_{k}=n$ and $m_{1}+m_{2}+\ldots+m_{k}=m$.

Remark that a H-type pseudoknots is a simple pseudoknot where $k=1$. Thus every H-type pseudoknot in $S$ is encoded by a subword $v=x^{n} y^{m} \bar{x}^{n} \bar{y}^{m}$. Finally, the following Proposition gives a way to encode any pseudoknotted structure where all pseudoknots are simple by a subset of the Dyck language with three kinds of pairs of parentheses, that is on the alphabet $\{d, \bar{d}, x, \bar{x}, y, \bar{y}\}$.

Proposition 1 Let $S$ be a pseudoknotted structure, and $w$ be the word on $\{d, \bar{d}, x, \bar{x}, y, \bar{y}\}$ that encodes $S$. Then $w$ can be encoded by a word on the alphabet $\{d, \bar{d}, x, \bar{x}, y, \bar{y}\} \cup\{p, \bar{p}\}$ where every subword $v=x^{n} y^{m_{1}} \bar{x}^{n_{1}} y^{m_{2}} \bar{x}^{n_{2}} \ldots y^{m_{k}} \bar{x}^{n_{k}} \bar{y}^{m}$, corresponding to a H-type pseudoknot is replaced with $v^{\prime}=$ $p x^{n-1} y^{m_{1}} \bar{y}^{m_{1}} \bar{x}^{n_{1}} y^{m_{2}} \bar{y}^{m_{2}} \bar{x}^{n_{2}} \ldots y^{m_{k}} \bar{y}^{m_{k}} \bar{x}^{n_{k}-1} \bar{p}$.

In particular, every subword $v=x^{n} y^{m} \bar{x}^{n} \bar{y}^{m}$ corresponding to a simple pseudoknot is replaced with $v^{\prime}=p x^{n-1} y^{m} \bar{y}^{m} \bar{x}^{n-1} \bar{p}$.

Proof (sketch): The proof is straightforward, as there is an immediate one-to-one correspondance between the two kinds of words below. The transformation is illustrated in Figure 4(a) and Figure 4(b) respectively, for simple pseudoknots and for the particular case of H-type pseudoknots. 

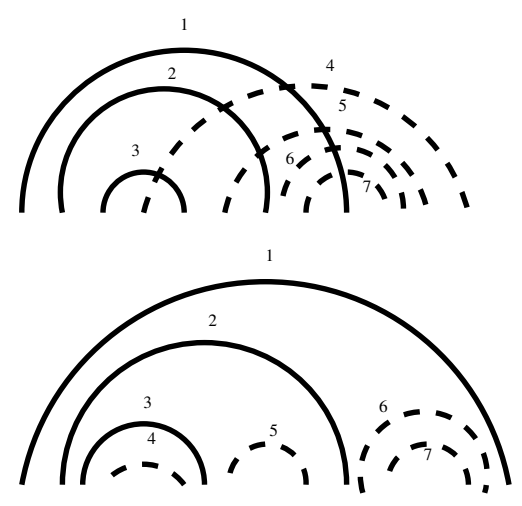

(a) Simple pseudoknot
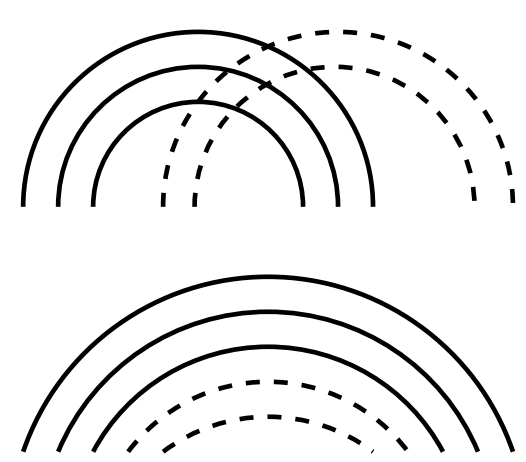

(b) H-type pseudoknot

Fig. 4: Top: two pseudoknots. Bottom: their encodings by Proposition 1

\subsection{Asymptotic results.}

For each of the D\&P, A\&U, R\&G, and $C \& C$ classes, we give an asymptotic equivalent for the number of structures of size $n$. In each case, the proof is in three steps: (i) We design an unambiguous context-free grammar which generates the language that encodes the considered structures, according to Proposition 1. (ii) From the grammar, we deduce an algebraic equation satisfied by the ordinary generating function (o.g.f.) of the language. (iii) From this equation, we compute an asymptotic formula for the number of structures of size $n$. For any class $X \& Y$, we write $X \& Y(n)$ for its number of structures having $n$ arcs.

\subsubsection{The Akutsu-Uemura class (A\&U).}

Following [1, 3], the A\&U structures are composed of non crossing edges and of any number of simple pseudoknots (Fig. 11). As these pseudoknot can embed other substructures which can be pseudoknotted in turn, they are said to be recursive [1].

\section{Theorem 2}

$$
A \& U(n)=\frac{\alpha_{1}}{2 \sqrt{\pi}} \omega_{1}^{n} n^{-3 / 2}(1+O(1 / n)),
$$

where $\alpha_{1}=0.6575407644 \ldots, \omega_{1}=7.547308334 \ldots$, are algebraic constants.

Proof: Let $L_{A \& U}$ be the language that encodes the A\&U class, according to Proposition 1 . The following unambiguous context-free grammar generates $L_{A \& U}$ :

$$
S \rightarrow d S \bar{d} S|P ; P \rightarrow p S X \bar{p} S| \epsilon ; X \rightarrow x S X \bar{x} S Y|y Y S \bar{y} S ; Y \rightarrow y S Y \bar{y} S| \epsilon
$$

The two rules in the first line allow to generate non crossing arcs and to put pseudoknots anywhere. The other rules generate words that correspond to the code for a simple pseudoknot.

Given the grammar, we obtain the set of recursive equations for the o.g.f. of the various sets defined in the 1-to-1 encoding. Letting the formal symbol $z$ denote an arc, we thus have through a straightforward translation:

$S(z)=z S^{2}(z)+P(z) ; P(z)=z S^{2}(z) X(z)+1 ; X(z)=z S^{2}(z) Y(z)(X(z)+1) ; Y(z)=z S^{2}(z) Y(z)+1$ 
By iterated bottom-up substitutions, we ultimately get that the o.g.f. $S(z)$ is solution of the algebraic equation

$$
F(z, S)=z^{2} S^{4}-2 z S^{3}+z S^{2}+S-1=0,
$$

from which we can derive the number of structures of size $n$.

For this proof we present in some details the main steps of the computations that have to be performed in order to get the asymptotics for an o.g.f. given by the algebraic implicit equation $F(z, S)=0$ satisfied by the o.g.f. $S(z)$.

Since $\partial F /\left.\partial z\right|_{z=0, S=1}=1$ is defined and $\partial F /\left.\partial S\right|_{z=0, S=1}=1$ is non vanishing, $z=0$ is not a singular point for $S$; by the implicit function theorem, $S(z)$ exists as a regular function in a circular neighborhood of $z=0$ until $\partial F / \partial S$ vanishes. The radius of convergence $\rho_{1}$ of the o.g.f. $S(z)$ is thus a solution of the system $\{F(z, S)=0, \partial F / \partial S(z, S)=0\}$. At such a point the local holomorphic solution $z=\zeta(S)$ is no longer invertible, which implies that this point is a singular point for the o.g.f. $S(z)$. The Darboux method allows to get accurate information and precise asymptotics for the Taylor expansion of $S(z)$.

Let $\left(z=\rho_{1}, S=\sigma_{1}\right)$ be the point of the Riemann surface of the solution located on the fold issued from $(z=0, S=1)$, which is closest to $(z=0, S=1)$ and for which $\partial F / \partial S=0$. This point is usually unique and located on the positive real axis, since the o.g.f. is indeed a function of $z$ with all coefficients being positive. At this point, the local expansion of $z$ with respect to $S$ writes:

$$
z=\rho_{1}+\frac{1}{2} \frac{d^{2} z}{d S^{2}}\left(S-\sigma_{1}\right)^{2}+\frac{1}{3 !} \frac{d^{3} z}{d S^{3}}\left(S-\sigma_{1}\right)^{3}+\ldots,
$$

since the first derivative, $\frac{d z}{d S}=-\frac{\partial F / \partial S}{\partial F / \partial z}$ vanishes at $\left(z=\rho_{1}, S=\sigma_{1}\right)$ and the second derivative $\frac{d^{2} z}{d S^{2}}=$ $-\frac{\partial^{2} F / \partial S^{2}}{\partial F / \partial z}$ does not.

Hence taking the square root of the previous equation we get the Taylor expansion at $\left(z=\rho_{1}, S=\sigma_{1}\right)$ :

$$
\sqrt{1-z / \rho_{1}}=\beta_{1}\left(S-\sigma_{1}\right)+\beta_{2}\left(S-\sigma_{1}\right)^{2}+\ldots,
$$

with $\beta_{1}=-\sqrt{\frac{1}{2} \frac{\partial^{2} F / \partial S^{2}}{\partial F / \partial z}}$, which can now be inverted locally giving:

$$
S=\sigma_{1}-\sqrt{\frac{2 \rho_{1} \partial F /\left.\partial z\right|_{z=\rho_{1}, S=\sigma_{1}}}{\partial^{2} F /\left.\partial S^{2}\right|_{z=\rho_{1}, S=\sigma_{1}}}} \sqrt{1-z / \rho_{1}}+O\left(1-z / \rho_{1}\right) .
$$

The expansion can be calculated at any order, so that we obtain for the coefficients $A \& U(n)$ an infinite asymptotic development whose dominant term is given by the first square root in the previous expansion, since it is well-known that $\left[z^{n}\right]-\sqrt{1-z / \rho}=\frac{1}{2 \sqrt{\pi}} \rho^{-n} n^{-3 / 2}(1+O(1 / n))$ :

$$
\left[z^{n}\right] S(z)=\sqrt{\frac{2 \rho_{1} \partial F /\left.\partial z\right|_{z=\rho_{1}, S=\sigma_{1}}}{\partial^{2} F /\left.\partial S^{2}\right|_{z=\rho_{1}, S=\sigma_{1}}}} \frac{1}{2 \sqrt{\pi}} \rho_{1}^{-n} n^{-3 / 2}(1+O(1 / n)) .
$$

We thus get the general form of the solution, as stated in the theorem, with $\alpha_{1}=\sqrt{\frac{2 \rho_{1} \partial F /\left.\partial z\right|_{z=\rho_{1}, S=\sigma_{1}}}{\partial^{2} F /\left.\partial S^{2}\right|_{z=\rho_{1}, S=\sigma_{1}}}}$ and $\omega_{1}=1 / \rho_{1}$. In order to get the values for the constants in the expansions and for the radius of convergence, we used Maple. From Equation 1 , we compute the partial derivatives $\partial F / \partial z=2 z S^{4}-$ 
$2 S^{3}+S^{2}$ and $\partial F / \partial S=4 * z^{2} * S^{3}-6 * z * S^{2}+2 * z * S+1$. The system is too complex to be solved formally; so we lower the degree in $S$ by considering the combination $R=4 F-S \partial F / \partial S=-2 z S^{3}+2 z S^{2}+3 S-4$ which has to vanish at the points where $F$ and $\partial F / \partial S$ do. Since $R$ is of degree 1 in $z$, it is easy to get an expression for $z$ that we substitute into $\partial F / \partial S$, obtaining that $8 S^{3}-31 S^{2}+42 S-20$ should equivalently be zero. Hence we obtain 3 possible algebraic roots, one being real $\sigma_{1}$ and the other two conjugate complex numbers. Only $\sigma_{1}=1.403556586 \ldots$ and the associated real value of $z$ for which $F(z, S)=0-\rho_{1}=0.1324975681 \ldots-$ are of interest. A direct approximate solution using the floating point solver of Maple confirms this situation and a more involved study or the Riemann surface also yields $\rho_{1}=0.1324975681 \ldots$ to be the radius of convergence of the series. Further computations provide all the constants encountered in the proof and stated in the theorem.

\subsubsection{The Dirks and Pierce class (D\&P).}

Structures of D\&P class are characterized by the presence of non crossing edges and any number of $\mathrm{H}$-type pseudoknots [4, 3].

\section{Theorem 3}

$$
D \& P(n)=\frac{\alpha_{2}}{2 \sqrt{\pi}} \omega_{2}^{n} n^{-3 / 2}(1+O(1 / n)),
$$

where $\alpha_{2}=0.7534777262 \ldots, \omega_{2}=7.3148684640 \ldots$, are algebraic constants.

Proof (sketch): The following unambiguous grammar generates the language that encodes the D\&P structures, according to Proposition 1 .

$$
S \rightarrow d S \bar{d} S|P ; P \rightarrow p X S \bar{p} S| \epsilon ; X \rightarrow x S X \bar{x} S|y S Y \bar{y} S ; Y \rightarrow y S Y \bar{y} S| \epsilon
$$

ù From this grammar, we get the following algebraic equation:

$$
F(z, S)=z^{3} S^{6}-z^{2} S^{5}+2 z S^{3}-z S^{2}-S+1=0
$$

which is very similar to the equation satisfied by the o.g.f. for the $A \& U$ family. We solve it in the same way, and find out the dominant singularity in $z=\rho_{2}=0.1367078581 \ldots, S=\sigma_{2}=1.439796009 \ldots$, with the same local behaviour, implying similar asymptotics for the coefficients. The only problem encountered in finding this dominant singularity comes from the fact that there exists another singularity closer to the origin in $z=\mu=0.08794976637 \ldots, S=\tau=7.169944393 \ldots$, but which is not on the same fold of the Riemann surface and which therefore does not have to be taken into consideration.

\subsubsection{The Reeder ang Giegerich class (R\&G).}

It corresponds to the structures of Reeder and Giegerich's algorithm [15]. It has a $\mathcal{O}\left(n^{4}\right)$ complexity.

\section{Theorem 4}

$$
R \& G(n)=\frac{\alpha_{3}}{2 \sqrt{\pi}} \omega_{3}{ }^{n} n^{-3 / 2}(1+O(1 / n)),
$$

where $\alpha_{3}=1.165192913 \ldots, \omega_{3}=6.576040092 \ldots$, are algebraic constants. 
Proof: In [15], the following grammar is given (we removed the unpaired bases):

$$
S \rightarrow S S|d S \bar{d}| x^{k} S y^{l} S \bar{x}^{k} S \bar{y}^{l} \mid \epsilon
$$

This grammar is not context-free. However, we remark that the pseudoknot defined here is a particular case of a H-Type pseudoknot. So by applying Proposition 1 again, we define the following context free grammar:

$$
S \rightarrow d S \bar{d} S|P ; P \rightarrow p X \bar{p} S| \epsilon ; X \rightarrow x X \bar{x}|S y Y \bar{y} S ; Y \rightarrow y Y \bar{y}| S
$$

Computations as above lead to the result.

Additionally, the following theorem places this new class into Condon et al.'s classification.

Theorem $5 R \& G \subset D \& P, L \& P \cap R \& G \neq \emptyset$ and $R \& G \not \subset L \& P$

Proof (sketch): The grammar wich describes the pseudoknots in $\mathrm{R} \& \mathrm{G}$ is less general than the grammar for H-type pseudoknots. So $R \& G \subset D \& P$ and $L \& P \cap R \& G \neq \emptyset$. As R\&G structures can contain several pseudoknots, we have $L \& P \cap R \& G \neq L \& P$.

\subsubsection{The Cao and Chen class (C\&C).}

It corresponds to the structures of Cao and Chen's algorithm [2], whose complexity is $\mathcal{O}\left(n^{6}\right)$.

Theorem 6

$$
C \& C(n)=\frac{\alpha_{4}}{2 \sqrt{\pi}} \omega_{4}^{n} n^{-3 / 2}(1+O(1 / n)),
$$

where $\alpha_{4}=1.665071176 \ldots, \omega_{4}=5.856765093 \ldots$, are algebraic constants.

Proof (sketch): The following non context-free grammar generates the C\&C structures:

$$
S \rightarrow S S|d S \bar{d}| x^{k} S y^{l} \bar{x}^{k} S \bar{y}^{l} \mid \epsilon
$$

It can be translated into a context-free grammar which is a restriction of the $\mathrm{R} \& \mathrm{G}$ grammar:

$$
S \rightarrow d S \bar{d} S|P ; P \rightarrow p X \bar{p} S| \epsilon ; X \rightarrow x X \bar{x}|S y Y \bar{y} S ; Y \rightarrow y Y \bar{y}| \epsilon
$$

Computations as above lead to the result.

Additionally, we easily state that

Theorem $7 C \& C \subset D \& P, L \& P \cap C \& C \neq 0, C \& C \not \subset L \& P$ and $C \& C \subset R \& G$

\subsubsection{The Lyngsø and Pedersen class (L\&P).}

We already gave a closed formula and an asymptotic equivalent for this class in Section 3 . It can be shown that its generating series can also be found in a very simple way by designing a context-free grammar. This will not be developed in this extended abstract. 


\section{Conclusion}

We proved that most classes of pseudoknotted structures that can be predicted by exact algorithms (all but R\&E for which the problem remains open) can be encoded by context-free languages. We extended Condon et al.'s hierarchy by adding two more classes, and we computed closed or asymptotic formulas for the cardinality of all classes but one.

These results, summarized in Table 1, allow us to quantify the relationship between the complexity of an algorithm and the generality of the class that it can handle. Notably, from a strict quantitative point of view, the growth of complexity by a factor $n^{2}$ between the PKF and L\&P classes seems not to be justified compared to the very small increase in cardinality. The situation is even worse for the $\mathrm{C} \& \mathrm{C}$ class, whose related algorithm has a stronger complexity than the $\mathrm{R} \& \mathrm{G}$ one, while $C \& C \subset R \& G$ and the ratio of their cardinalitues is exponential. On the other hand, the linear increasing between PKF andR\&G complexities seems very reasonable compared to the exponential increasing of the cardinalities.

Besides, the fact that most of the classes are encoded by context-free languages gives an easy way to perform uniform or controlled non uniform random generation of pseudoknotted RNA structures, with standard methods and tools (see e.g. [6, 5, 14]).

Acknowledgements. This research was supported in part by the ANR project BRASERO ANR-06BLAN-0045, and by the Digiteo project "RNAomics".

\section{References}

[1] T. Akutsu. Dynamic programming algorithms for RNA secondary structure prediction with pseudoknots. Discrete Applied Mathematics, 104:45-62, 2000.

[2] S. Cao and S-J Chen. Predicting structured and stabilities for h-type pseudoknots with interhelix loop. RNA, 15:696-706, 2009.

[3] A. Condon, B. Davy, B. Rastegari, S. Zhao, and F. Tarrant. Classifying RNA pseuknotted structures. Theorical computer science, 320:35-50, 2004.

[4] N.A. Dirks, R.M. Pierce. A partition function algorithm for nucleic acid secondary structure including pseudoknots. J Comput Chem, 24:1664-1677, 2003.

[5] P. Duchon, P. Flajolet, G. Louchard, and G. Schaeffer. Boltzmann samplers for the random generation of combinatorial structures. Combinatorics, Probability, and Computing, 13(4-5):577-625, 2004. Special issue on Analysis of Algorithms.

[6] Ph. Flajolet, P. Zimmermann, and B. Van Cutsem. A calculus for the random generation of labelled combinatorial structures. Theoretical Computer Science, 132:1-35, 1994.

[7] I. L. Hofacker, P. Schuster, and P. F. Stadler. Combinatorics of RNA secondary structures. Discr. Appl. Math, 89, 1996.

[8] F. W. D. Huang and M. Reidys. Statistics of canonical RNA pseudoknot structures. Journal of Theoretical Biology, 253(3):570-578, 2008. 
[9] E. Y. Jin and C. M. Reidys. RNA pseudoknot structures with arc-length $\geq 3$ and stack-length $\geq \sigma$. Discrete Appl. Math., 158(1):25-36, 2010.

[10] W.A. Lorenz, Y. Ponty, and P. Clote. Asymptotics of RNA shapes. Journal of Computational Biology, 15(1):31-63, Jan-Feb 2008.

[11] R. B. Lyngsø and Pedersen C. N. RNA pseudoknot prediction in energy based models. Journal of computational biology, 7:409-428, 2000.

[12] M. E. Nebel. Combinatorial properties of RNA secondary structures. Journal of Computational Biology, 9(3):541-574, 2003.

[13] R. Nussinov, G. Pieczenik, J. R. Griggs, and Kleitman D. J. Algorithms for loop matching. SIAM J. Appl. Math., 35:68-82, 1978.

[14] Y. Ponty, M. Termier, and A. Denise. GenRGenS: Software for generating random genomic sequences and structures. Bioinformatics, 22(12):1534-1535, 2006.

[15] J. Reeder and R. Giegerich. Design, implementation and evaluation of a practical pseudoknot folding algorithm based on thermodynamics. BMC Bioinformatics, 5:104, 2004.

[16] E. Rivas and S. R. Eddy. A dynamic programming algorithm for RNA structure prediction including pseudoknots. Journal of molecular biology, 285:2053-2068, 1999.

[17] E. A. Rødland. Pseudoknots in RNA secondary structures: representation, enumeration, and prevalence. Journal of Computational Biology, 13(6):1197-1213, 2006.

[18] N. J. A. Sloane and Simon Plouffe. The Encyclopedia of Integer Sequences. Academic Press, 1995.

[19] W.T. Tutte. A census of planar maps. Canadian Journal of Mathematics, 15:249-271, 1963.

[20] Y. Uemura, A. Hasegawa, S. Kobayashi, and T. Yokomori. Tree adjoining grammars for RNA structures prediction. Theorical computer science, 210:277-303, 1999.

[21] M. Vauchaussade de Chaumont and X.G. Viennot. Enumeration of RNA's secondary structures by complexity. In V. Capasso, E. Grosso, and S.L. Paven-Fontana, editors, Mathematics in Medecine and Biology, volume 57 of Lecture Notes in Biomathematics, pages 360-365, 1985.

[22] G. Vernizzi, H. Orland, and A. Zee. Enumeration of RNA structures by matrix models. Phys. Rev. Lett., 94:168103, 2005.

[23] T. R. S. Walsh and A. B. Lehman. Counting rooted maps by genus. iii: Nonseparable maps. $J$. Combinatorial Theory Ser. B, 18:222-259, 1975.

[24] M. S. Waterman. Secondary structure of single-stranded nucleic acids. Advances in Mathematics Supplementary Studies, 1(1):167-212, 1978.

[25] M. Zucker and P. Stiegler. Optimal computer folding of large RNA sequences using thermodynamics and auxiliary information. Nucleic Acid Research, 9:133-148, 1981. 